\title{
Light Curve and Spectral Evolution of Type IIb Supernovae
}

\author{
Anjasha Gangopadhyay ${ }^{1,4 *}$, Kuntal Misra ${ }^{1}$, Andrea Pastorello ${ }^{2}$, \\ Devendra Kumar Sahu ${ }^{3}$, Mridweeka Singh ${ }^{1,4}$, Raya Dastidar ${ }^{1}$, \\ Gadiyara Chakrapani Anupama ${ }^{3}$, Brijesh Kumar ${ }^{1}$, Shashi Bhushan Pandey ${ }^{1}$
}

${ }^{1}$ Aryabhatta Research Institute of observational sciencES, Nainital, 263002, India

${ }^{2}$ INAF - Astronomical Observatory of Padova, Italy

${ }^{3}$ Indian Institute of Astrophysics, Bangalore, Karnataka, 560034, India

${ }^{4}$ Pt. Ravi Shankar Shukla University, Raipur, 492001, India

\begin{abstract}
Stripped-Envelope Supernovae constitute the sub-class of core-collapse supernovae that strip off their outer hydrogen envelope due to high stellar winds or due to interaction with a binary companion where mass transfer occurs as a result of Roche lobe overflow. We present here the photometric and spectroscopic analysis of a member of this class : SN 2015as classified as a type IIb supernova. Light curve features are similar to those of SN $2011 \mathrm{fu}$ while spectroscopic features are quite similar to those of SN 2008ax and SN $2011 \mathrm{dh}$. Early epoch spectra have been modelled with SYN++ which indicates a photospheric velocity of 8500 $\mathrm{km} \mathrm{sec}^{-1}$ and temperature of $6500 \mathrm{~K}$. Spectroscopic lines show transitioning from $\mathrm{H}$ to He features confirming it to be a type IIb supernova. Prominent oxygen and calcium emission features are indicative of the asymmetry of the ejecta. We also estimate the signal to noise ratio of the $3.6 \mathrm{~m}$ telescope data. This telescope is located at ARIES, Devasthal, Nainital at an altitude of $2450 \mathrm{~m}$. We also show the comparison plots of spectra taken with a $2 \mathrm{~m}$ and $4 \mathrm{~m}$ class telescopes to enlighten the importance of spectral features displayed by bigger diameter telescopes.
\end{abstract}

\section{Introduction}

Stripped envelope core-collapse supernovae (SE-SNe) constitute a composite group of supernovae (SNe) that result from death of massive stars having masses greater than $8 \mathrm{M}_{\odot}$ and strip off their outer hydrogen and helium envelopes due to high stellar wind or interaction with a companion star in a binary system, where mass transfer occurs as a result of Roche lobe overflow (Podsiadlowski et al. 1992). By short stripped SNe (Clocchiatti \& Wheeler 1997), we refer to the group of type IIb, Ib, Ic and broad lined Ic named as Ic-BL. Type IIb SNe provide a direct link between type II and I SNe in the way that they transition from H-rich to H-deficient SNe.

Light curves of type IIb SNe usually show an initial peak, then rapid decline and again rise to a secondary maximum. Initial peak is due to shock breakout and secondary peak is due to thermalisation of $\gamma$ rays and decay of ${ }^{56} \mathrm{Ni} \rightarrow{ }^{56} \mathrm{Co} \rightarrow{ }^{56} \mathrm{Fe}$ while some SNe may lack the primary peak. Spectra show transitions from H-rich to H-deficient features. Also, prominent doublets due to OI 6300, $6364 \AA$, Ca 7291, $7324 \AA$ and singlet

\footnotetext{
*anjasha@aries.res.in, anjashagangopadhyay@gmail.com
} 
like Mg $4571 \AA$ and triplet Ca NIR features could be seen in the nebular spectra. Type IIb SNe provide a direct link between type II and I SNe. However, we lack a clear knowledge regarding the connection between the $\mathrm{SNe}$ and their stellar progenitors. Some of the open questions in this field include : a. How stars get rid of their outer envelopes before exploding ? ; is it through strong winds, like eruptions in luminous blue variables, by mass transfer to a close binary companion or any other mechanism come into play ? (Langer 2012). Is there a distinct separation between type IIb and type $\mathrm{Ib} \mathrm{SNe}$ or is there a continuity related by some layer of Hydrogen ? (Heger et al. 2003). Both theory and observations can help to predict the exact nature and hence to provide a more physically relevant subdivision of SE-SNe types.

\section{OBSERVATIONS AND DATA REDUCTION}

Prompt photometric follow-up of SN 2015as was carried out a few days after discovery using the 104-cm Sampurnanand Telescope (ST) (Sagar 1999), ARIES Nainital, 130-cm Devasthal Fast Optical Telescope (DFOT) (Sagar et al. 2012), ARIES Nainital, 182-cm Ekar Asiago Telescope, Italy and 200-cm Himalayan Chandra Telescope (HCT) (Prabhu \& Anupama 2010), IAO, Hanle. The imaging observations were done in Johnson Cousins Bessel B, V, R, I filters and SDSS u, g, r, i, z filters. Photometric monitoring of SN 2015as started on 17 Nov, 2015 and continued upto 30 June, 2016. 24 epochs of medium resolution optical spectra of SN 2015as were obtained using the 182-cm Ekar Asiago telescope and 200-cm Himalayan Chandra Telescope from 17 Nov, 2016 to 23 June, 2016. Necessary pre-processing was done with standard tasks in IRAF whereas data were reduced using DAOPHOT.

\section{RESULTS AND DISCUSSIONS}

\subsection{Photometric Features}

Complete multi-band light curve evolution of SN 2015as is illustrated in Fig. 1. Also, the comparison plot of SN 2015as with other members of the sub-class is shown in Fig. 2. It can be seen that the early time light curve of SN 2015as matches quite well with SN 2011fu, but they are faster than all other members of the sub-class implying that there is less $\gamma$ ray trapping and a very small amount of positrons being deposited in the SN ejecta. The decline in magnitude 15 days post maximum $\triangle m_{15}$ in $\mathrm{B}$ and $\mathrm{V}$ bands are $\triangle m_{15}(\mathrm{~B})=1.62 \pm 0.024$ and $\triangle m_{15}(\mathrm{~V})=0.99 \pm 0.03$.

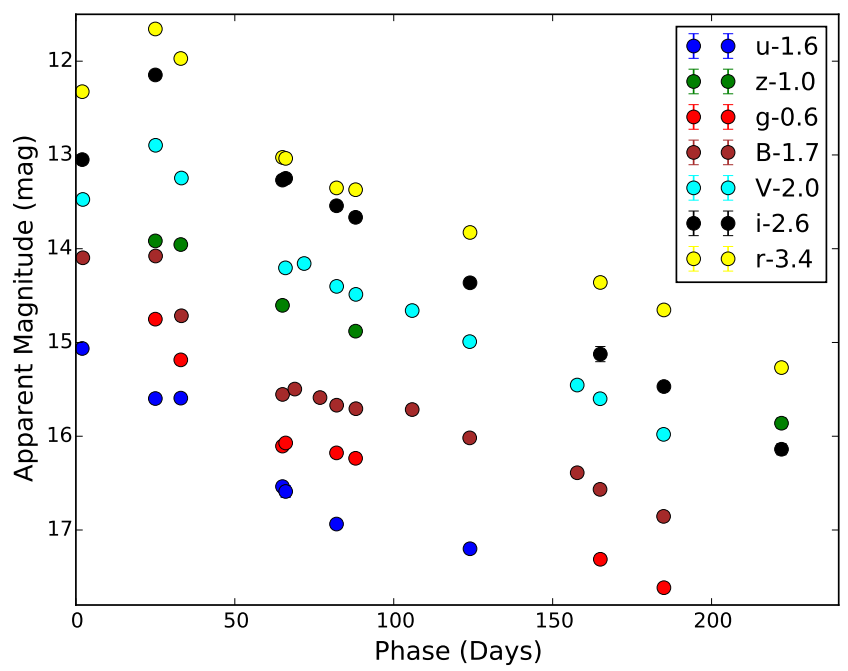

Figure 1: Multi-band light curve evolution of $\mathrm{SN}$ 2015as. Here phase refers to days since discovery.

The light curves indicate that even though B and V match well with other members of the sub-class, the $\mathrm{R}$ and I band light curves show a flattening similar to that of SN 2011fu. It could be inferred that the light 
curves of SNe IIb decline faster than the normal decay rates of ${ }^{56} \mathrm{Co} \rightarrow{ }^{56} \mathrm{Fe}\left(0.0098\right.$ mag day $\left.^{-1}\right)$ indicating inefficient $\gamma$ ray trapping by the SN ejecta. The absolute magnitude of SN 2015as in V-band is $\mathbf{M}_{v}=-16.89$ mag, assuming a distance to the host galaxy of $19.2 \mathrm{Mpc}$ and $\mathrm{E}(\mathrm{B}-\mathrm{V})=0.008 \mathrm{mag}$. It is important to note that SN 2015as lies in the fainter end of the group of stripped envelope supernovae.

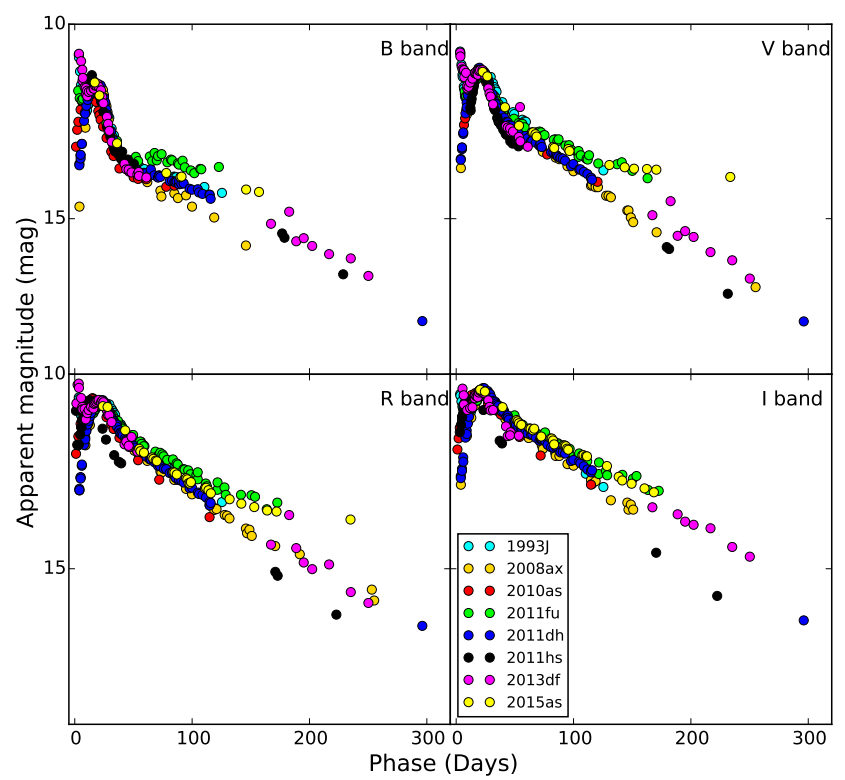

Figure 2: Comparison plot of SN 2015as with other members of the subclass. Here phase refers to days since discovery.

\subsection{Spectroscopic Features}

Fig. 3 shows the transition of spectroscopic features for SN 2015as. The 1st spectrum shows prominent Balmer lines while the 2 nd spectrum shows prominent $\mathrm{He}$ features. The spectrum also marks the beginning of $\mathrm{Ca}$ NIR triplet, $\mathrm{Ca} \mathrm{H} \& \mathrm{~K}$ features have still not vanished. We also estimate the signal to noise ratio for the $3.6 \mathrm{~m}$ telescope data which is tabulated in Table 1 . The $\mathrm{S} / \mathrm{N}$ ratio is estimated using the standard diameter, f number, dark current of the $3.6 \mathrm{~m}$ telescope. Assuming a FWHM of 2 arcsec and exposure time of $300 \mathrm{sec}$, magnitudes are varied in the range 15-25 for the complete $\mathrm{U}$ to I band which indicate that supernovae beyond 20 mag are fairly detectable with the $3.6 \mathrm{~m}$ telescope. Fig. 4 shows the comparison plot of spectra of SN 2015as taken with a $2 \mathrm{~m}$ class telescope at an epoch of 120 days post dicovery compared with SN 1993J taken with a 4m class telescope taken at a very closeby epoch. Both the supernova have magnitudes comparable at this epoch and the $4 \mathrm{~m}$ spectra shows prominent $\mathrm{Ti}$, Ba lines which are not visible in the $2 \mathrm{~m}$ class spectra.

\section{CONCLUSIONS}

SN 2015as is a type IIb supernova which transitioned from type II to type I during the period of our observations. Light curve features are very similar to SN $2011 \mathrm{fu}$, decay rates are also comparable to SN $2011 \mathrm{fu}$ while spectral features showed transitions from being a $\mathrm{H}$ rich to a He dominant supernova. Also, SN beyond 20 mag can be detected with a fairly good signal to noise ratio. Nebular phase spectra taken with comparable magnitudes show prominent lines of $\mathrm{Ti}$ and $\mathrm{Ba}$ in a $4 \mathrm{~m}$ class telescope which is absent in the $2 \mathrm{~m}$ class spectra. Thus, with $4 \mathrm{~m}$ class telescopes, we can probe deeper into the ejecta and also possibly blended lines could easily be traced out through bigger diameter telescopes. 


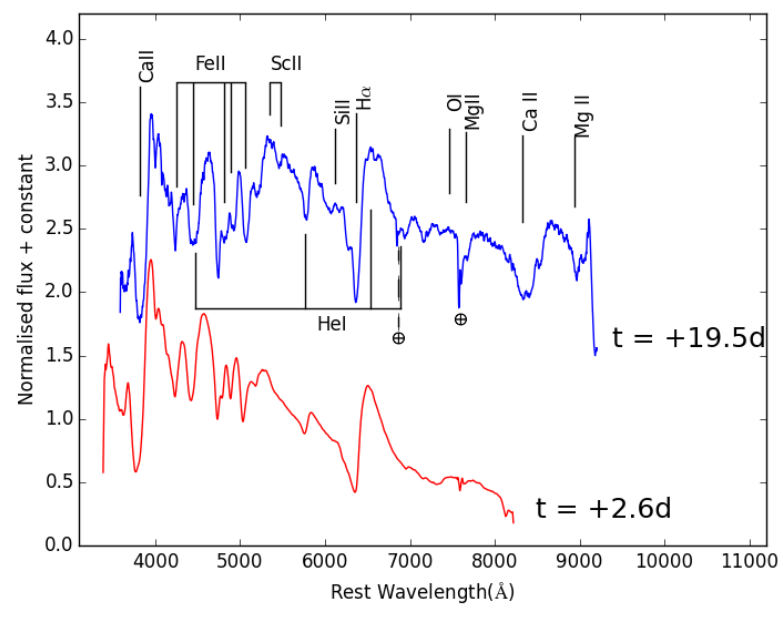

Figure 3: Comparison plot of the SN 2015as spectrum obtained with a $2 \mathrm{~m}$ class telescope.

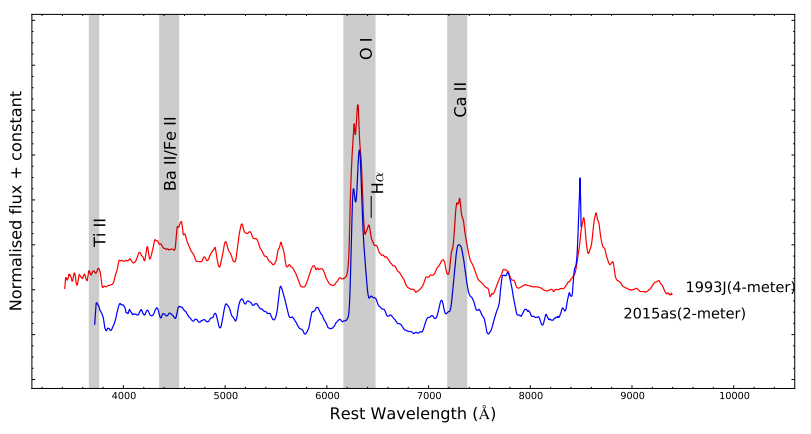

Figure 4: Comparison plot of the SN 1993J spectrum obtained with a $4 \mathrm{~m}$ class telescope.

Table 1: Estimated Signal to Noise Ratio of the data to be obtained by the $3.6 \mathrm{~m}$ telescope :

\begin{tabular}{|c|ccccc|}
\hline Mag & $\mathrm{U}$ & $\mathrm{B}$ & $\mathrm{V}$ & $\mathrm{R}$ & $\mathrm{I}$ \\
\hline 15.0 & 612.2 & 1751.9 & 1019.6 & 929.3 & 513.1 \\
16.0 & 248.5 & 727.4 & 415.4 & 373.8 & 205.2 \\
17.0 & 99.7 & 294.8 & 167.0 & 149.5 & 81.9 \\
18.0 & 39.8 & 118.2 & 66.7 & 59.6 & 32.6 \\
19.0 & 15.9 & 47.2 & 26.6 & 23.7 & 13.0 \\
20.0 & 6.3 & 18.8 & 10.6 & 9.5 & 5.2 \\
21.0 & 2.5 & 7.5 & 4.2 & 3.8 & 2.1 \\
22.0 & 1.0 & 3.0 & 1.7 & 1.5 & 0.8 \\
23.0 & 0.4 & 1.2 & 0.7 & 0.6 & 0.3 \\
24.0 & 0.2 & 0.5 & 0.3 & 0.2 & 0.1 \\
25.0 & 0.1 & 0.2 & 0.1 & 0.1 & 0.1 \\
\hline
\end{tabular}




\section{Acknowledgements}

One of the authors AG thanks Lina Tomasella for providing the data of $182 \mathrm{~cm}$ Ekar Asiago Telescope, Italy. We thank the observing staff and observing assistants at $104 \mathrm{~cm} \mathrm{ST}$ and $130 \mathrm{~cm}$ DFOT. We also thank the $201 \mathrm{~cm}$ HCT staff for their support during observations of SN 2015as.

\section{References}

Podsiadlowski P., Joss P. C, Hsu J. J. 1992, ApJ, 391, 246

Clocchiatti A., Wheeler J. C. 1997, ApJ, 491, 357C

Langer N. 2012, ARA\&A, 50, 107

Heger A., Fryer C. L., Woosley S. E. et al. 2003, ApJ, 591, 288

Sagar R. 1999, Current Science, 77, 643 Prabhu T. P., Anupama G. C., 2010, in Astronomical Society of India Conference Series

Sagar R., Kumar B., Omar A., Pandey A. K. 2012, in Ground-based and Airborne Telescopes IV. p. 84441T (arXiv:1304.2474), doi:10.1117/12.925634 